\title{
Las relaciones económicas y comerciales entre China y Argentina en la era de Mauricio Macri ${ }^{1}$
}

\author{
The economic and trade relations between \\ China and Argentina in the era of Mauricio Macri
}

Lin $\mathrm{Hua}^{2}$

\begin{abstract}
After his assumption to the President of Argentina, Mauricio Macri quickly adopted a series of measures of economic, social and diplomatic reform, showing his different concept and model of governance from previous governments. Affected by this, the bilateral relations between China and Argentina have undergone some notable changes. This article analyzes the development of Sino-Argentine relations in the last two years by dividing it into four phases, making a diagnosis of these changes, and presenting opportunities and challenges that face the relations of both countries. This article argues that the development of the economic and trade relations between China and Argentina has entered a new historical stage and there will be more possibilities for further development of bilateral cooperation in the future. The diversified cooperation is an indispensable way for the two countries to continue to foster bilateral relations, but this requires detailed study and constant innovation in terms of contents, models and mechanisms.
\end{abstract}

Palabras clave: Macri; China; Argentina; iniciativa de la Franja y la Ruta

Resumen: Después de asumir la presidencia de la Argentina, Mauricio Macri adoptó rápidamente una serie de medidas de reforma económica, social y diplomática, mostrando un modelo diferente de gobernanza en referencia a los gobiernos anteriores. Afectadas por esto, las relaciones bilaterales entre China y Argentina han experimentado algunos cambios notables. Este trabajo realiza un análisis del desarrollo de las relaciones sino-argentinas en los últimos dos años, visualizándolo en 4 fases bien diferenciadas. Este artículo sostiene que el desarrollo de las relaciones sino-argentinas ha entrado en una nueva etapa histórica, con la perspectiva de que siga desarrollándose la cooperación bilateral en el futuro. La cooperación diversificada es una forma indispensable para que los dos países sigan impulsando las relaciones bilaterales, pero requiere el estudio detallado y la innovación constante en términos de contenidos, modelos y mecanismos.

Keywords: Macri; China; Argentina; one belt and one road Initiative

1 Recibido: 2/11/2917. Aceptado: 5/11/2017.

2 Licenciada en economía. Profesora asociada del Instituto de América Latina, Academia China de Ciencias Sociales. Secretaria general del Centro de Estudios Argentinos del Instituto. 


\section{Introducción}

Mauricio Macri, líder del partido político Propuesta Republicana (Pro), de orientación centro- derechista, asumió el cargo de presidente de la Argentina el 10 de diciembre de 2015, terminando con un período de doce años de gobiernos de izquierda. Después de su asunción, el presidente Macri adoptó rápidamente una serie de medidas de reformas económicas, sociales y diplomáticas, que implicaron un modelo y concepto de gobernanza diferente en referencia a los gobiernos anteriores. Afectadas por esto, las relaciones bilaterales entre China y Argentina han experimentado algunos cambios notables, que son resultados necesarios del ajuste de política interior y exterior de Argentina. Este artículo tiene como objetivo analizar estos cambios y su impacto en las relaciones futuras entre los dos países, así como sinterizar cuáles son las oportunidades y obstáculos que enfrentan las relaciones bilaterales durante la administración de Macri. El trabajo está compuesto por 5 partes. En la primera, se analizan 4 fases del desarrollo de las relaciones sino-argentinas en los últimos dos años. En la segunda, se hace un diagnóstico de los cambios de las relaciones bilaterales. En la tercera y cuarta se presentan respectivamente oportunidades y desafíos que enfrentan las relaciones entre ambas partes, para finalizar con algunas conclusiones. Como se adelantó, el desarrollo de las relaciones sinoargentinas ha entrado en una nueva etapa histórica y habrá más posiblidades para que siga desarrollándose la cooperación bilateral en el futuro, pero requiere el estudio detallado y la innovación constante en términos de los contenidos, modelos y mecanismos.

\section{El desarrollo de relaciones sino-argentinas desde la asunción del presidente de Mauricio Macri}

Desde la asunción del presidente Macri, las relaciones bilaterales entre China y Argentina han pasado por algunos altibajos. De acuerdo con su desarrollo y sus cambios, así como con varios puntos nodales, se puede dividir en cuatro fases.

La primera fase fue desde la asunción de Macri hasta marzo de 2016. Durante este período, el gobierno de Macri se acercó activamente a los Estados Unidos y a los países europeos, mostrando una clara tendencia a alejarse de China. Macri no estaba de acuerdo con los modelos cooperativos que se habían desarrollado entre los gobiernos anteriores y China, y propuso en varias ocasiones reconsiderar los acuerdos de cooperación firmados por el gobierno anterior, lo que llevó a mantener en suspenso a una serie de grandes proyectos. En marzo, un barco pesquero chino se hundió después de ser atacado por la policía marítima argentina. Este suceso generó especulaciones y dudas sobre el futuro desarrollo de las relaciones entre China y Argentina.

La segunda fase la ubicamos entre los meses de abril a agosto de 2016. Este es un "período de rodaje". O sea, tanto China como Argentina trataban de adaptarse al nuevo contexto. Durante este período, China y Argentina fortalecieron sus diálogos de alto nivel. El presidente chino Xi Jinping se reunió con Macri en la IV Cumbre de Seguridad Nuclear, realizada en abril en Washington, y los dos gobiernos reiteraron la voluntad común de fomentar la cooperación. La ministra de Relaciones Exteriores de Argentina, Susana Malcorra, visitó dicho país en mayo y llegó a un acuerdo con China sobre la profundización de la cooperación estratégica. Desde entonces, varios ministros de Argentina realizaron visitas al país asiático para lograr un conocimiento profundo sobre la cooperación en distintos secto- 
res. Al mismo tiempo, el nuevo gobierno argentino fue adquiriendo gradualmente una comprensión integral sobre la cooperación sino-argentina, lo cual se visualizó en la reevaluación de los proyectos de cooperación firmados por ambas partes.

La tercera fase comenzó en septiembre de 2016 y finalizó en mayo de 2017. Luego de la interacción contínua y los diálogos ininterrumpidos, las relaciones sinoargentinas volvían a entrar en un desarrollo estable. A principios de septiembre de 2016, Macri participó en la Cumbre del G20 celebrada en China y tuvo un nuevo encuentro con el presidente Xi. Aprovechando esta oportunidad, los dos países firmaron los acuerdos de cooperación modificados.

La cuarta fase se inicia después del mes de mayo de 2017. A partir de ese momento, China y Argentina establecieron un nuevo lazo de cooperación: la iniciativa de "la Franja y la Ruta". En este sentido, en mayo de 2017, el presidente Macri, fue uno de los dos jefes de Estado latinoamericanos invitados y viajó a China nuevamente para asistir a la Cumbre de "la Franja y la Ruta" en carácter de visita de Estado. Durante esa visita, ambos gobiernos suscribieron 16 acuerdos de cooperación económicos y comerciales por unos 17 mil millones de dólares estadounidenses. En junio, el Banco Asiático de Inversión en Infraestructura anunció la aprobación del ingreso de la Argentina, que se convirtió en uno de los seis Estados miembros latinoamericanos.

\section{Diagnóstico sobre las relaciones sino-argentinas después de la llegada de Macri al poder}

En el año 2016, las relaciones sino-argentinas tuvieron dificultades temporales. Pero, en líneas generales, no estuvieron fuera de la senda del desarrollo anterior. Es decir, la llegada de Macri al poder significó una alternancia política normal de Argentina y tuvo poco impacto en el desarrollo holístico de las relaciones bilaterales.

En primer lugar, las relaciones sino-argentinas cuentan con una buena base de desarrollo. Desde el establecimiento de las relaciones diplomáticas entre ambos países, las relaciones bilaterales se han desarrollado establemente y no han existido conflictos ni confrontaciones graves entre ambas partes. Los dos países comparten puntos de vista similares sobre muchos temas internacionales importantes y tienen intereses y aspiraciones comunes en estos asuntos. En los últimos años, los dos gobiernos han prestado gran importancia al desarrollo de las relaciones bilaterales, y han realizado frecuentes visitas de alto rango. En 2004 ambos Estados establecieron la asociación estratégica, y en 2014 la elevaron al nivel de asociación estratégica integral. China respalda el reclamo argentino por la soberanía de las Islas Malvinas, mientras que Argentina defiende el principio de "una sola China". Las bases y fundamentos arriba mencionados no han cambiado en la era de Macri.

En segundo lugar, China y Argentina siguen siendo importantes socios económicos y comerciales. No han cambiado las tres correlaciones importantes de dichos intercambios: la complementariedad, el beneficio mutuo y la interdependencia. En las áreas económico-comerciales ambos países se atraen, se necesitan, se apoyan y se benefician mutuamente, lo que no sólo sienta las bases para generar una tendencia de desarrollo de más colaboración y menos competencia, más consenso y menos confrontación, sino que también proporciona un espacio más amplio y de mayores oportunidades para el desarrollo futuro de las relaciones bilaterales. Pero vale la pena señalar que el motor del 
desarrollo de las relaciones económicas y comerciales sino-argentinas se ha transformado. Duante la primera década de este siglo, el comercio bilateral era el impulsor predominante de esas relaciones. Pero en los últimos años, la inversión y la cooperación financiera se han convertido en otros dos motores muy importantes.

En tercer lugar, las dificultades y fricciones en las relaciones sino-argentinas han sido normales en el desarrollo de las relaciones entre Estados. Las relaciones diplomáticas ya tienen una historia de 45 años, pero la profundización de las relaciones bilaterales se ha producido en los últimos 15 años. Durante los primeros 30 años, tras el establecimiento de las relaciones diplomáticas, los intercambios políticos, económicos y comerciales entre ambas partes fueron limitados. Aunque no había conflicto, ni contradicción, ni tensión, faltaba la oportunidad para tener una comprensión mutua profunda. En los últimos 15 años, surgieron las fricciones comerciales y la discrepancia. Pero éstas generaron más oportunidades para intercambiar y comprenderse mutuamente, impulsando a la vez la profundización de las relaciones bilaterales en el proceso que denominamos: de "fricción - ajuste - consenso".

Finalmente, el cambio en la política argentina hacia China después de la llegada al poder de Macri, es resultado de su implementación de la diplomacia pragmática y multilateral. En comparación con el gobierno anterior, el ajuste diplomático de Macri es obvio. Tanto el mejoramiento de los vínculos con Europa y los Estados Unidos, como el acercamiento a la Alianza del Pacífico son reflejos del pragmatismo y del equilibrio diplomático. A largo plazo, la diplomacia multilateral es beneficiosa para el desarrollo de las relaciones entre China y Argentina, proporcionando un contexto internacional multilateral favorable y creando más espacios y posibilidades de cooperación.

\section{Oportunidades para las relaciones bilaterales}

Desde 2003 a 2013, el comercio bilateral aumentó casi cinco veces. Pero después de 2014, el intercambio entre ambos países tuvo altibajos. Esto muestra que las actuales relaciones económicas y comerciales han entrado en un nuevo período histórico, necesitando nuevos impulsos y estímulos, que provendrán de las oportunidades creadas durante los próximos dos años del gobierno de Macri.

En primer lugar, el constante desarrollo de la urbanización de China y la continua expansión de la clase media brindarán más oportunidades para las exportaciones argentinas hacia China. En los últimos años, la desaceleración del crecimiento económico del país asiático ha sido motivo de preocupación y de debate de los académicos a nivel internacional. Algunos analistas entienden que la disminución de la demanda china de recursos naturales ha causado la caída de los precios de las materias primas a nivel mundial. Sin embargo otra opinión considera que a medida que China continúa su urbanización, su demanda general de recursos naturales sigue siendo muy fuerte. En este artículo se sostiene que a pesar de que la desaceleración del crecimiento económico de China reduce en cierta medida la demanda externa de algunos productos, no ha generado gran impacto negativo en aquellos países que son proveedores de productos agrícolas hacia el país asiático, tal es el caso de Argentina. Para explicar este tema, partimos de la base en primer lugar, de que el avance de la urbanización china no se ha detenido. De 2001 a 
2015, la tasa de urbanización aumentó del $38 \%$ al $55 \%,{ }^{3}$ lo que significa que la demanda del país asiático de productos agrícolas aumentará constantemente. Por otro lado, los grupos de ingresos medios de China están surgiendo. En algunas metropólis como Beijing, Shanghai y Guangzhou, la demanda de los residentes por bienes de consumo de mejor calidad y de alta gama se está incrementando aceleradamente. De hecho, esto brinda la oportunidad para el ingreso de más productos argentinos al mercado chino, especialmente productos agroindustriales de alta calidad.

En segundo lugar, la cooperación entre China y Argentina tiene plataformas de cooperación cada vez más amplias. Como se mencionó anteriormente, el comercio bilateral ha sufrido un cuello de botella luego de 10 años de rápido crecimiento. Esto tiene que ver con la disminución de los precios de productos básicos. En otras palabras, la cooperación económica y comercial ya no puede depender únicamente del comercio tradicional. Es necesario crear nuevos espacios y plataformas de cooperación. Mientra que el G20, el Banco Asiático de Inversión en Infraestructura, y la iniciativa de "la Franja y la Ruta" proporcionan más posibilidades de cooperación multilateral para el desarrollo de las relaciones económicas entre ellos. Como miembros del G20, China y Argentina prestan gran importancia a este mecanismo. Los dos países asisten activamente a la Cumbre, procurando aprovechar este mecanismo para participar en la gobernanza mundial, buscar soluciones a los problemas encontrados en su propio desarrollo económico y social, así como exponer las reivindicaciones y expresar opiniones, en nombre de los países en desarrollo. En cuanto a la iniciativa de "la Franja y la Ruta", después de la visita de Macri a China en mayo de 2017, Argentina ha comenzado a pensar cómo alinear esa iniciativa con la estrategia de desarrollo de los países de América Latina, sobre todo de la Argentina. Macri propuso que la iniciativa china colabore con la Iniciativa de la Integración de la Infraestructura Regional Sudamericana (IIRSA) para conectar Asia y los países a lo largo de "la Franja y la Ruta" con su región. Si esta idea se puede poner en práctica, promoverá en gran medida la cooperación sino-argentina en el campo de la infraestructura. Además, Argentina, a través del ingreso al Banco Asiático de Inversión en Infraestructura, también tendrá más oportunidades de cooperación con China y los países a lo largo de "la Franja y la Ruta", especialmente en el sector financiero.

En tercer lugar, el actual gobierno de Argentina hace hincapié en el papel del mercado y la eficiencia, y reduce la intervención del gobierno y el proteccionismo, con el fin de transformar la economía argentina hacia un modelo liberal y más abierto a la inversión extranjera, lo que ayudará a mejorar el ambiente de inversión y de negocios, proporcionando más oportunidades para que las empresas chinas amplien sus inversiones en Argentina. Por otra parte, después de una recesión de dos años, se espera que la macroeconomía argentina vuelva a crecer en 2017, lo que también contribuirá a fortalecer la confianza y la voluntad de los inversores chinos.

Finalmente, el nuevo modelo de cooperación podrá inyectar nueva vitalidad a las relaciones sino-argentinas. En mayo de 2017, en la víspera de la visita de Macri a Beijing, el Sr. Jack Ma, el fundador del Grupo Alibaba (la mayor empresa de Internet de China y la plataforma del comercio electrónico a nivel global), visitó Argentina, y tuvo el segundo encuentro con el presidente Macri tras la Cumbre del G-20 en septiembre de 2016 en 
Hangzhou. Posteriormente, el gobierno argentino y Alibaba firmaron un acuerdo de cooperación estratégica, lo que indica que el comercio electrónico transfronterizo probablemente se convierta en un nuevo modelo de cooperación económica y comercial entre China y Argentina. En la actualidad, la demanda de los consumidores chinos por los productos de alta gama está creciendo cada día más, y se acostumbran a hacer compras en internet, lo cual es muy favorable para el ingreso de los productos de buena calidad argentinos al mercado chino. Ciertamente, un grupo de empresarios argentinos cree que tal cooperación no es factible debido a la falta de apoyo legal y técnico necesario. Pero en el contexto en que la economía mundial y el uso de Internet se estrechan cada vez más, sin duda la plataforma de comercio electrónico será una nueva opción para la cooperación sino-argentina, que no sólo promoverá el desarrollo de las exportaciones hacia China y el desarrollo de las PYMEs de la Argentina, sino también fomentará una serie de infraestructuras, tales como logística local y pago electrónico, entre otros. Todo esto se podrá acelerar si el gobierno argentino adoptase políticas de apoyo relevantes para concretar este objetivo.

\section{Dificultades y desafíos}

Los desafíos siempre coexisten con las oportunidades. En este sentido, ambos gobiernos deben considerar la realidad. Estos desafíos o dificultades no son nuevos y seguramente permanecerán durante todo el período del gobierno de Macri.

Primero, el conocimiento mutuo entre los dos países está seriamente ausente. Aunque los intercambios en los últimos años son cada vez más importantes, aún falta un entendimiento verdadero, especialmente "people to people". La distancia geográfica es un gran obstáculo que dificulta enormemente los intercambios entre los pueblos, incluidos los turistas. Precisamente debido a la distancia, los dos países carecen de un enlace histórico y legado de cooperación, como por ejemplo la inmigración a gran escala, etc. Además, el idioma es otro obstáculo difícil de superar. En China, la enseñanza del idioma español ha ocupado un lugar importante en las universidades, incrementándose cada vez más el interés por el idioma. Sin embargo, en Argentina, la enseñanza del idioma chino todavía no se ha popularizado. A pesar de que muchos estudiantes aprenden chino en los Institutos de Confucio, son limitados los que pueden hablar chino fluidamente. Por último, los canales de intercambio y comunicación entre pueblos son insuficientes. Aunque en Argentina se efectúa anualmente la fiesta de Año Nuevo chino, y el pueblo chino conoce el fútbol argentino y el tango, estos eventos sólo forman una pequeña parte de las culturas. Por estas razones, los vínculos entre ambos países en los campos político- económicos son cada vez más estrechos, pero los contactos sociales y los intercambios culturales son aún limitados, lo que genera una asimetría importante entre las relaciones. Debido al insuficiente intercambio ideológico y cultural, se producen malentendidos y preocupaciones sobre China y las relaciones sino-argentinas en el pueblo argentino, incluyendo algunos medios de comunicación, académicos y funcionarios.

Segundo, tanto las empresas chinas como las empresas argentinas, carecen de conocimientos y estudios sobre las condiciones nacionales y mercados de la otra parte. Debido a los escasos conocimientos sobre leyes y reglamentos, relaciones laborales, riesgos políticos, estándares ambientales, etc, las empresas chinas se encuentran con muchas dificultades para adaptarse al mercado argentino. Al mismo tiempo que las empresas 
argentinas no han realizado investigaciones profundas del mercado chino y de los hábitos y preferencias de consumidores, por eso no han aprovechado bien al mercado más grande del mundo, lo que ha llevado a que muchos productos argentinos de buena calidad no se hayan podido insertar al mercado chino.

Tercero, en cuanto al comercio bilateral, ya sea el incremento de la cantidad, o la mejora de la estructura de los bienes, se enfrentan a un desafío a largo plazo. Desde el punto de vista del valor comercial, según las estadísticas de la Aduana de China, el comercio bilateral en 2013 alcanzó un récord histórico de 14,8 mil millones de dólares. Pero en los 3 años posteriores, no ha podido superar esta cantidad. Además, Argentina viene registrando un déficit comercial con China desde el año 2011, ${ }^{4}$ lo cual genera una gran preocupación en Argentina. Pero cabe destacar que tanto en términos del valor comercial como el valor de déficit, existe una enorme diferencia entre las estadísticas chinas y argentinas (véase los cuadro 1 y 2 ).

Cuadro 1 Comercio bilateral entre China y Argentina según las estadísticas de la Aduana de China entre 2008-2016 (millones de dólares)

\begin{tabular}{|l|c|c|c|c|c|c|c|c|c|}
\hline año & $\mathbf{2 0 0 8}$ & $\mathbf{2 0 0 9}$ & $\mathbf{2 0 1 0}$ & $\mathbf{2 0 1 1}$ & $\mathbf{2 0 1 2}$ & $\mathbf{2 0 1 3}$ & $\mathbf{2 0 1 4}$ & $\mathbf{2 0 1 5}$ & $\mathbf{2 0 1 6}$ \\
\hline $\begin{array}{l}\text { Exportación } \\
\text { china }\end{array}$ & 5038 & 3483 & 6116 & 8504 & 7011 & 8750 & 7683 & 8813 & 7201 \\
\hline $\begin{array}{l}\text { Importación } \\
\text { china }\end{array}$ & 9358 & 4306 & 6802 & 6291 & 5864 & 6089 & 5247 & 5716 & 5119 \\
\hline $\begin{array}{l}\text { Balance } \\
\text { Comercial }\end{array}$ & -4320 & -819 & -686 & 2213 & 1147 & 2661 & 2436 & 3097 & 2082 \\
\hline
\end{tabular}

Fuente: Las estadísticas de la Aduana de China.

Cuadro 2 Comercio bilateral entre China y Argentina según las estadísticas del INDEC de Argentina entre 2008-2016 (millones de dólares)

\begin{tabular}{|l|c|c|c|c|c|c|c|c|c|}
\hline año & $\mathbf{2 0 0 8}$ & $\mathbf{2 0 0 9}$ & $\mathbf{2 0 1 0}$ & $\mathbf{2 0 1 1}$ & $\mathbf{2 0 1 2}$ & $\mathbf{2 0 1 3}$ & $\mathbf{2 0 1 4}$ & $\mathbf{2 0 1 5}$ & $\mathbf{2 0 1 6}$ \\
\hline Exportacion china & 7103 & 4843 & 7678 & 10612 & 9932 & 11341 & 10743 & 11776 & 10483 \\
\hline Importación china & 6354 & 3950 & 6117 & 6507 & 5379 & 5837 & 4792 & 5388 & 4661 \\
\hline Balance Comercial & 749 & 893 & -1561 & 4105 & 4553 & 5504 & 5951 & 6389 & 5822 \\
\hline
\end{tabular}

Fuente: Sitio web oficial del INDEC, fecha de consulta: 1 de octubre de 2017.

http://www.indec.gob.ar/nivel4_default.asp?id_tema_1=3\&id_tema_2=2\&id_tema_3=40

El déficit comercial de Argentina con China tiene mucho que ver con el desequilibrio de la estructura de bienes. Aunque las exportaciones argentinas de manufacturas hacia China pueden expandirse a través del aumento de la exportación de productos agroindustriales, debido a las ventajas comparativas existentes de ambas partes, será difícil cambiar en el corto plazo el patrón actual de comercio bilateral, es decir, China exporta bienes manufacturados e importa productos primarios.

Una realidad ineludible es que la soja ocupa una posición muy importante en el comercio entre los dos países, pero existe algún conflicto de interés en la industria de procesamiento de soja.

4 Según las estadísticas del INDEC de Argentina, el déficit de Argentina con China empezó desde el año 2008. 
China en los últimos 20 años, y Argentina en los últimos 10 años, han aumentado rápidamente la capacidad de crushing de soja. Actualmente, la capacidad de crushing de China ocupa el primer lugar en el mundo, alcanzando 430 mil de toneladas de soja por día. ${ }^{5}$ Mientras que el crushing de Argentina ya ha superado a los Estados Unidos, ocupando el segundo lugar en el mundo. Como la producción de soja de China es tan baja que no puede satisfacer la demanda, necesita importaciones de gran cantidad del grano de soja. Pero en comparación con porotos de soja, Argentina prefiere exportar aceite de soja a China. Porque el aceite de soja pertenece a un producto procesado con un valor agregado mucho mayor que el poroto de soja. Además, la soja es un recurso relevante en Argentina, mientras que el crushing de soja es una herramienta importante por la reindustrialización tomando esta ventaja natural. Esta estrategia china de importar porotos de soja, realizar el crushing de soja en el mercado doméstico y autoabastecerse del aceite de soja, genera conflictos. En la actualidad, tanto en China como en Argentina, existen problemas de exceso de capacidad de crushing, lo que presenta un desafío para el comercio de aceite de soja entre ambas partes. A corto plazo, esta confrontación continuará existiendo.

Cuarto, las actividades de inversión de China en Argentina han sufrido un dilema. Tomando la infraestructura como ejemplo, por un lado, el gobierno argentino respalda y estimula la inversión en dicho sector, procurando impulsar la economía mediante la construcción de infraestructura. En julio de 2016, el gobierno argentino emitió el "Plan Nacional de Transporte e Infraestructura ", que implica 33.200 millones de dólares para la construcción de infraestructura durante la administración de Macri. En septiembre del mismo año, el Ministro de Transporte de Argentina, Guillermo Dietrich, viajó a China para presentar los proyectos. Pero por otro lado, los proyectos de infraestructura ya existentes no han logrado un avance rápido. El Complejo hidroeléctrico Jorge Cepernic-Néstor Kirchner ha sufrido varias suspensiones. En agosto de 2017 el gobierno aprobó nuevamente su construcción. $Y$ otros proyectos, como la estación espacial y la central nuclear están cuestionadas e incluso rechazadas por un grupo de organizaciones sociales y algunos funcionarios locales, lo que indica que más adelante las empresas chinas que planean invertir en Argentina, tienen que hacer investigaciones más detalladas sobre las condiciones del mercado y las necesidades de las provincias, con el fin de seleccionar proyectos más adecuados.

\section{Conclusión}

Después de la asunción del presidente Macri, en el contexto del ajuste de la política exterior de Argentina, las relaciones sino-argentinas han tenido algunos cambios. Sin embargo, este artículo argumenta que a pesar de ellos, la buena base y las importantes correlaciones de las relaciones entre ambos países no se han transformado. Este es un factor significativo del continuo desarrollo de las relaciones sino-argentinas en la era de Macri. El vínculo entre ambas partes ha entrado en una nueva etapa, en la que los estí-

5 Lorena D'angelo, "El crushing de soja en Argentina y Estados Unidos: realidad y perspectivas", noviembre de 2016, http://www.lorenadangelo.com/el-crushing-de-soja-en-argentina-y-estados-unidos-realidady-perspectivas/, fecha de consulta: 1 de octubre de 2017. 
mulos provienen del comercio, la inversión y la cooperación financiera. En el contexto de que el comercio bilateral, tanto en términos del incremento de la cantidad como del mejoramiento en la estructura comercial, se enfrenta a los desafíos, la cooperación diversificada es una manera indispensable para que los dos países sigan impulsando las relaciones bilaterales. Este artículo sostiene que los contenidos, modelos y mecanismos de la cooperación entre China y Argentina, tienen mucho espacio y muchas posibilidades para seguir desarrollándose, pero necesitan del estudio más detallado y la innovación constante. En cuanto al desequilibrio comercial existente entre ambas partes, el proteccionismo no es un instrumento eficaz. Tanto China como Argentina deben tomar en consideración cómo elevar la capacidad exportadora de Argentina a través de aprovechar la cooperación diversificada, con el fin de mejorar esta asimetría.

Sin duda alguna, en el curso del desarrollo de las relaciones entre China y Argentina en los últimos años, han existido obstáculos y desafíos, que se presentarán durante la administración de Macri. Entre estos retos, el fortalecimiento del conocimiento y la comprensión mutuos, será una tarea a largo plazo, pero también es un requisito previo para superar todas las otras dificultades. Esto requiere no sólo esfuerzos conjuntos de los dos gobiernos, sino también del apoyo y la participación de más fuerzas sociales y civiles. Cabe destacar que las relaciones sino-argentinas en la era de Macri también enfrentarán más y mejores oportunidades que nunca. Si los dos países hacen un buen uso de estas oportunidades, la complementariedad mutua se extenderá del comercio hacia otros terrenos, tales como la inversión, las finanzas, la cultura, la educación, el deporte, el arte, la ciencia y tecnología, a fin de concretar el objetivo de "ganar-ganar".

\section{Bibliografía}

López, A. y Ramos, D. (2008), “A la sombra del gigante chino: hay lugar para la Argentina en el nuevo mundo?", Boletín Informativo Techint 326, mayo-agosto de 2008.

D'angelo, L. "El crushing de soja en Argentina y Estados Unidos: realidad y perspectivas", noviembre de 2016, http://www.lorenadangelo.com/el-crushing-de-soja-enargentina-y-estados-unidos-realidad-y-perspectivas/

Oviedo, D. (2015), "Argentina y China: los actores del comercio sojero y el flujo migratorio", Revista Nueva Sociedad, No.259, septiembre-octubre de 2015, p.117-128.

Oviedo, D. (2016), “Estudio preliminar de las relaciones argentino-chinas a principios del gobierno de Mauricio Macri", Jiexizhongguo, Análisis y Pensamiento Iberoamericano sobre China, segundo trimestre de 2016, p.4-28.

Bolinaga, L. (2015), "Política china en el Río de la Plata, ¿Asociación estratégica o nueva dependencia?", Revista Nueva Sociedad, No.259, septiembre-octubre de 2015, p.69-80.

Gransow, B. (2015), “Inversiones chinas en infraestructura: ¿una situación en la que todos ganan?", Revista Nueva Sociedad, No.259, septiembre-octubre de 2015, p.93105.

Bonilla Soria, A. y Milet García, P. (editores)(2015), China en América Latina y el Caribe: Escenarios estratégicos subregionales, San José, C.R., FLACSO, CAF, 2015. 